$$
\text { تأثير خطوط النقل خارج الخدمة المؤزضة على تنظيم مرحِّة قياس المسافة }
$$

الخلاصة

يتضمن هذا البحث دراسة وتحليل تأثير خطوط النقل خارج الخدمة المؤرضة على تنظيم مرحّلة قياس المسافة،

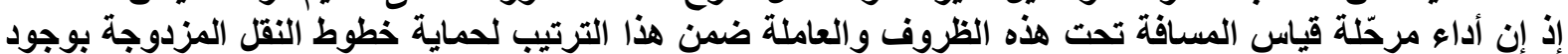

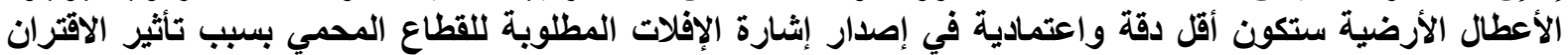

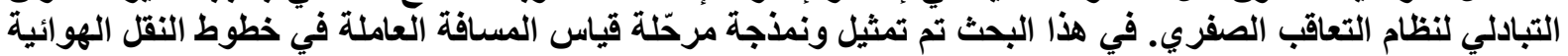
المزدوجة لشبكة كهرباء العراق للمنطقة الشمالية (INRG) باستخدام برنامج (Matlab\Simulink) لتحليل حالة فئة

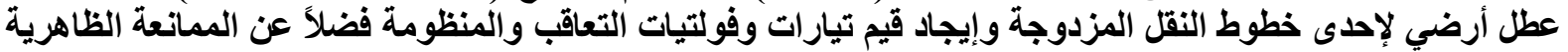

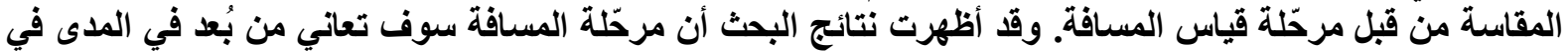

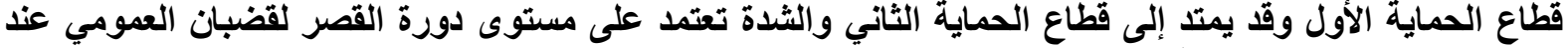
نهايتي خطوط النقل المزدوجة.

الكلمات الدالة: مرحّلة قياس المسافة، الاقتران التبادلي، موقع العطل.

\title{
Effect Of The Earthed Out of Service Transmission Lines On a Distance Measuring Relay Setting
}

\section{Dr. Abdul Ghani A. Abdul Ghafoor}

Electrical Department College of Engineering University of Mosul

\author{
Mr. Ahmed Ateya Al- \\ Badrani \\ Electrical Department \\ Institute of Mosul \\ Foundation of Technical Education
}

Abstract:

This paper includes a study and analysis of an earthed out of service for one of double circuit transmission lines impact on a Distance Measuring Relay (DMR) setting due to zero sequence system mutual coupling. The protection in a remaining line measures the fault impedance correctly, except when the line that is not in service is earthed at both ends. In this case a DMR performance which installed in this configuration at the ground faults occur is much less reliable and accuracy to get an ideal tripping protected zone for its. This study has been Modeling and simulting by using (MatlabiSimulink) program to analyze a steady state of ground fault on single circuit to calculate the DMR voltage,current and apparent impedance of sequences and system at various fault locations under operational status for the Iraqi North Regional Grids (INRG) $132 \mathrm{Kv}$ system. The paper results shows that mutual coupling by zero sequence current of earthed line may cause the DMR to seriously over-reach under different operational situations. The DMR first zone reach may extend to its second zone, So a DMR will give a trip signal for an external faults.

Keywords: Distance Measuring Relay, Mutual Coupling, Fault Location.

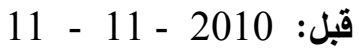

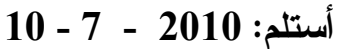




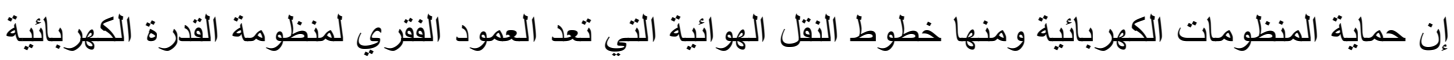

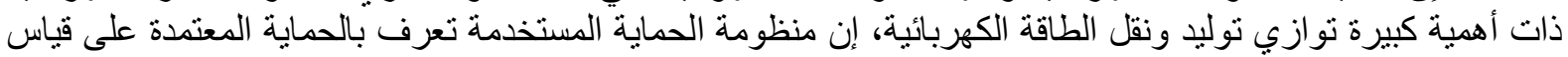

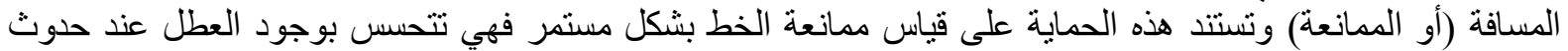

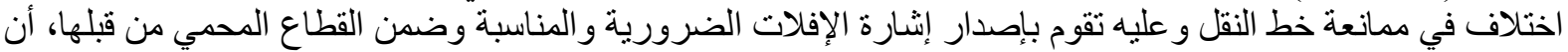

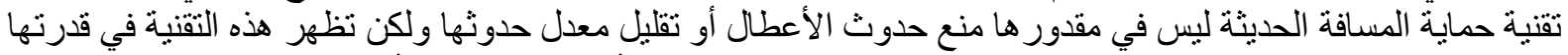

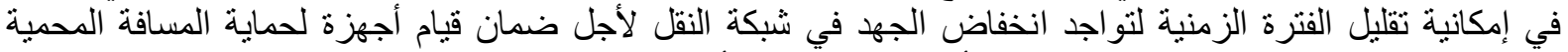

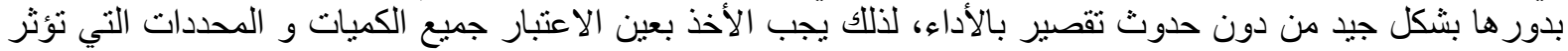

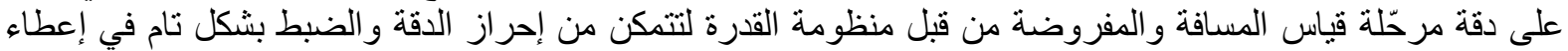

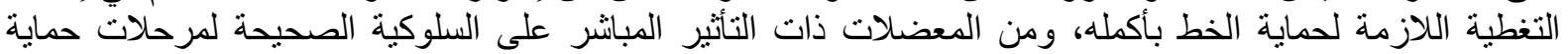

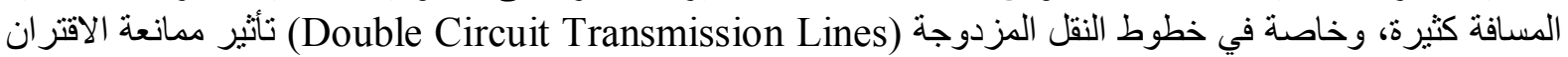
التبادلي بين خطوط النقل خارج الخدمة ( Out Of Service Lines) و المؤرضة لغرض الصيانة وباقي الخطوط

.[2],[1]

\section{تأثير الاقتران التبادلي في خطوط النقل الهوائية المزدوجة الدائرة:}

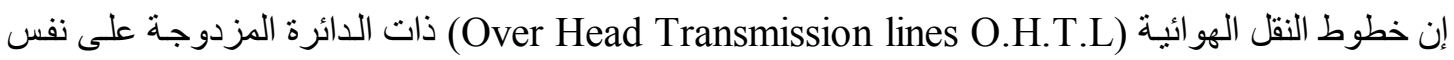

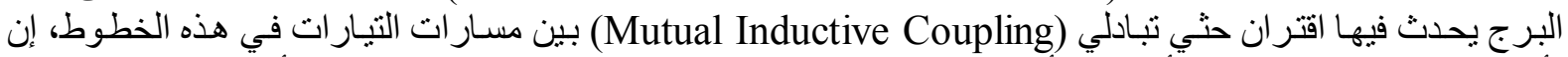

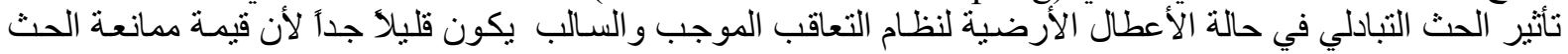

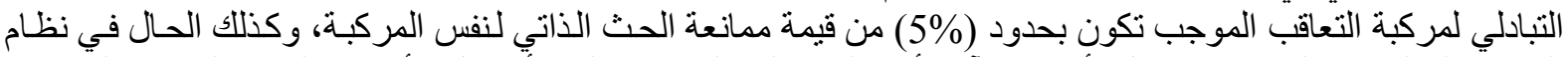

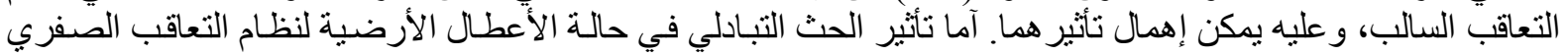

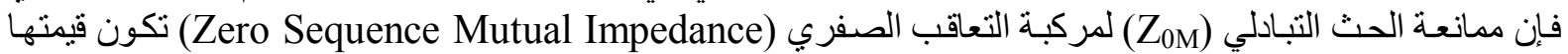

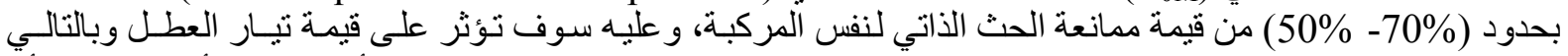

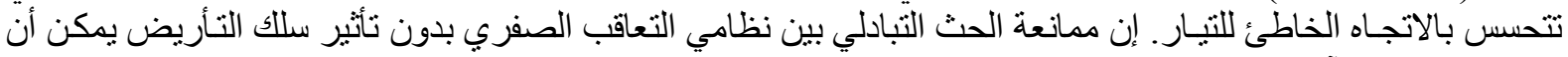
تعطى بالمعادلة الآتية [2],[3]

$$
\mathrm{Z}_{0 \mathrm{M}}=3 \cdot \mathrm{R}_{\mathrm{E}}+\left(\mathrm{j} . \omega \cdot 6 \cdot \ln \frac{D_{e}}{A_{L}}\right) \cdot 10^{-4} \quad[\Omega / \mathrm{km}]
$$

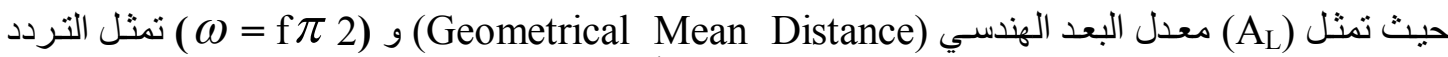

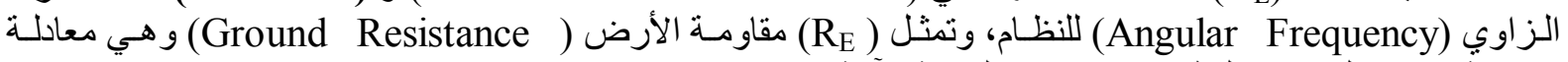
تجريية تعتمد على تردد النظام وتكون وفق العلاقة الآتية [2]:

$$
\mathrm{R}_{\mathrm{E}}=(\pi / 2) \cdot \omega \cdot 10^{-4} \quad[\Omega / \mathrm{km}]
$$

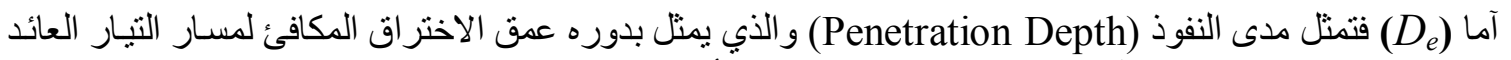

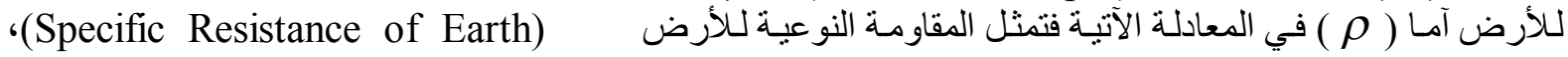

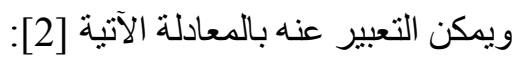

$$
D_{e}=1650 \sqrt{\frac{\rho}{\omega}}
$$

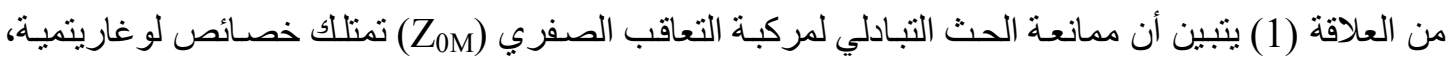

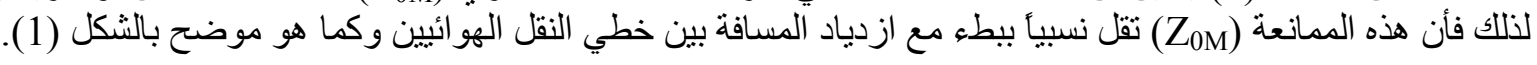

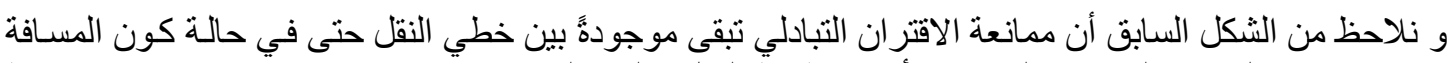

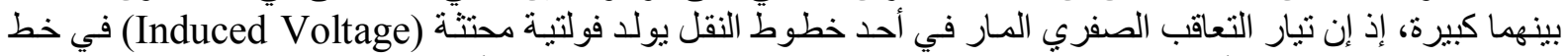

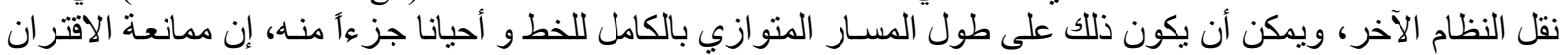

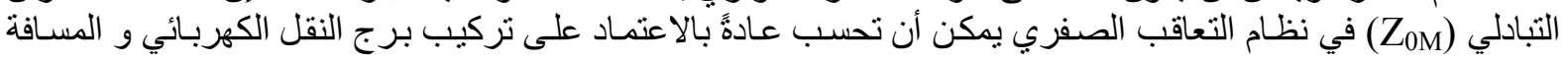




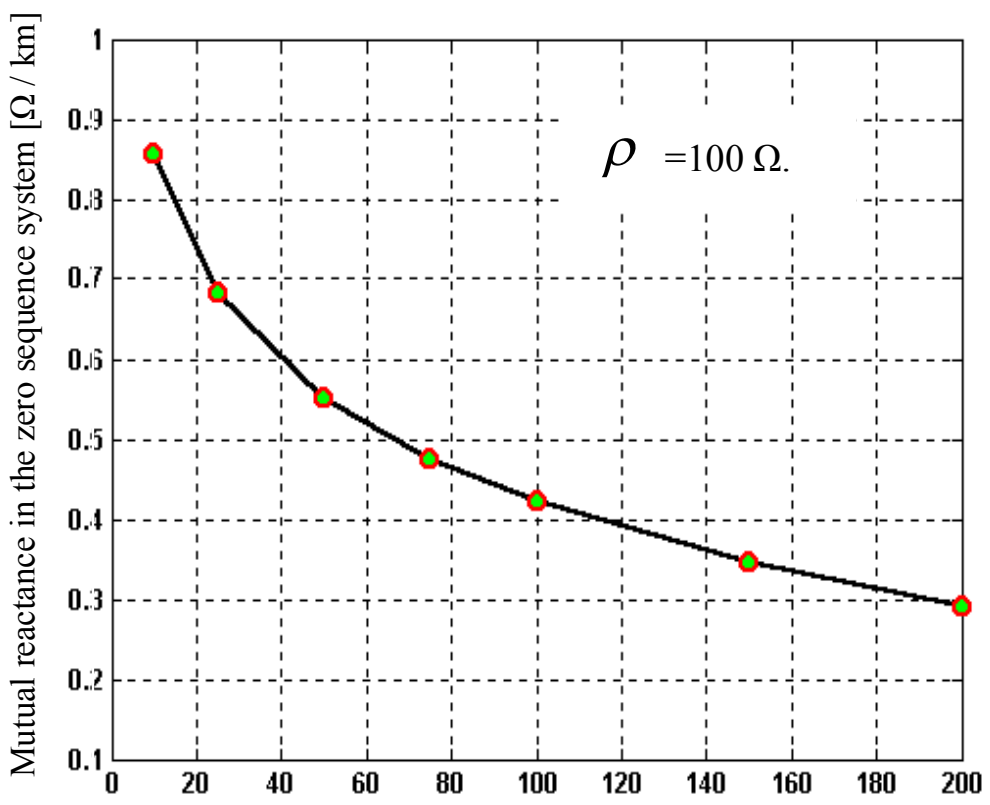

The distance between two over head T.L [m]

الثكل (1): تأثير مفاعلة الاقتران التبادلي لنظام النعاقب الصفري في خطوط النقل

\section{ظروف تثنغيل مرحّلة قياس المسافة في خطوط النقل الهوائية المزدوجة:}

من العو امل المؤثرة على نتائج قياس الممانعة بين موقع العطل وموقع المرحّلة هي ظاهرة المغناطيسية التبادلية

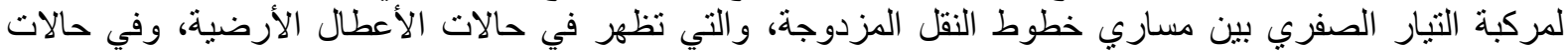

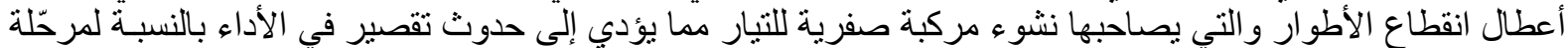

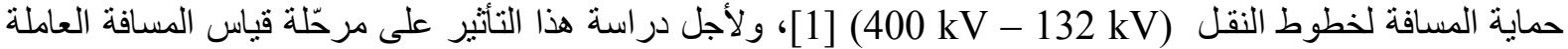

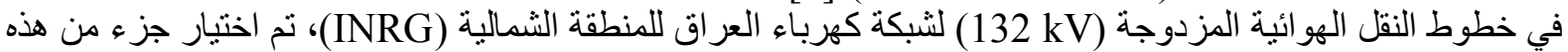

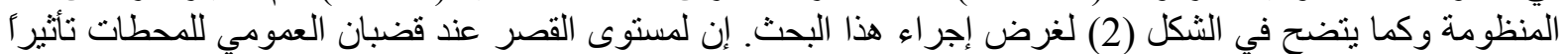

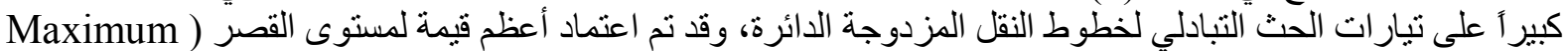
(Short Circuit Level



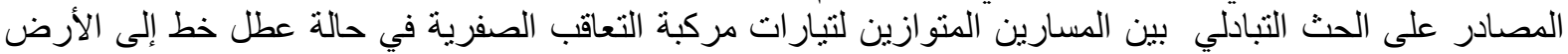

.[4](Line-Ground Fault)

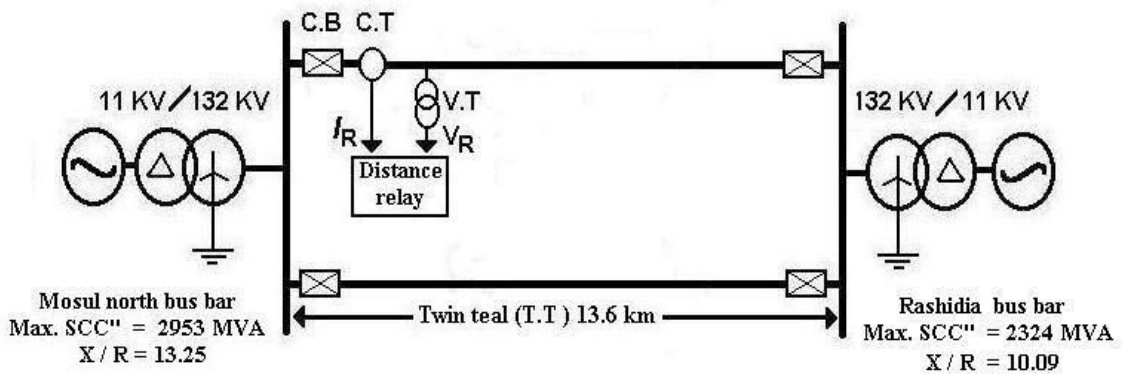

الثكل (2): جز ء من خطوط النقل العاملة بجه 132 kV) لثبكة كهرباء العر اق للمنطقة الثمالية

في هذا البحث تم أخذ حالة عطل طور مع الأرض في مسار الدائرة الأولى (A) لخط نقل الهو ائي المزدوج بينما

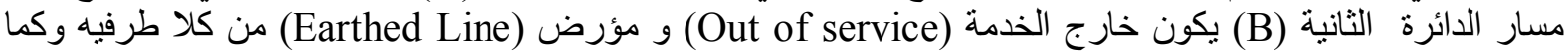

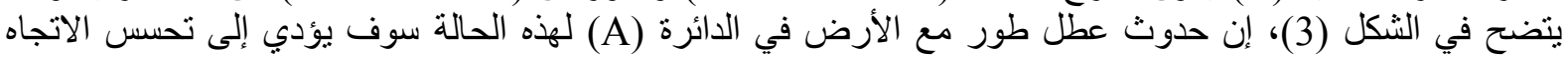


الخاطئ لتيار العطل بسبب نشوء مركبة صفرية للتيار، وبالتالي يؤدي إلى حدوث حث تبادلي بين نظامي التعاقب الصفري

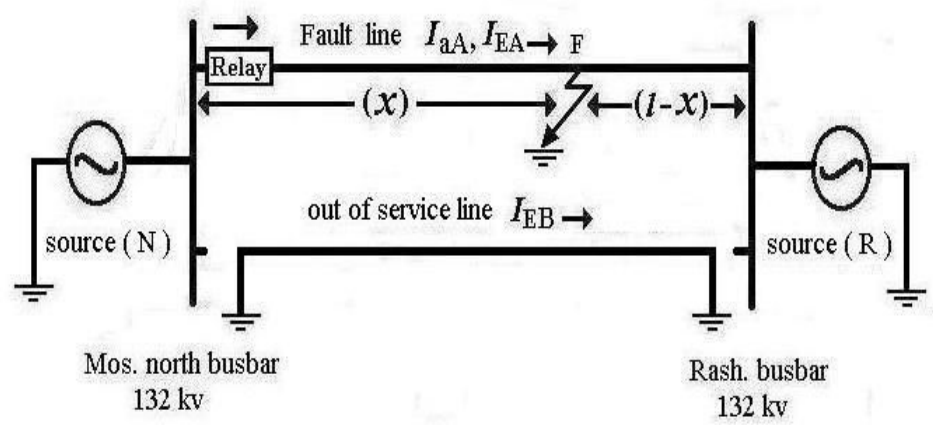

الثكل (3): يبين حالة حدوث عطل أرضي لمسار الدائرة الأولى (A) لخط نقل مزدوج

$$
\text { مع كون مسار الدائرة الثانية (B) خارج الخدمة و مؤرض }
$$

و لأجل تبيان هذه الظاهرة يجب تحليل وحساب الممانعة الظاهرية التي تقيسها مرحّلة قياس المسافة من خلال التهل

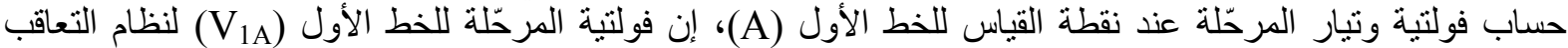

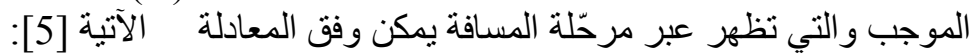

$\mathrm{V}_{1 \mathrm{~A}}=\mathrm{X} \cdot \mathrm{Z}_{1 \mathrm{~L}} \cdot I_{1 \mathrm{~A}}+\mathrm{V}_{1 \mathrm{~F}}$

وكذلك الحال بالنسبة لفولتية المرحّلة (V2A) نظام التعاقب السالب للمرحّلة وكالآتي [5]:

$\mathrm{V}_{2 \mathrm{~A}}=\mathrm{X} . \mathrm{Z}_{2 \mathrm{~L}} \cdot I_{2 \mathrm{~A}}+\mathrm{V}_{2 \mathrm{~F}}$

حيث تمثل (X) موقع العطل بالكيلومتر وتيار التعاقب الموجب للمرحّلة يكون مساويأ لتيار التعاقب السـالب لها

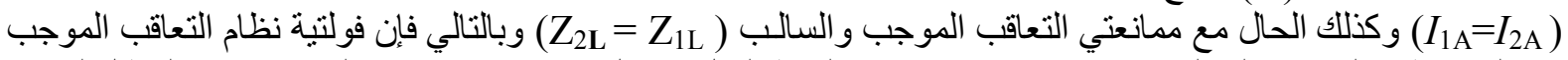

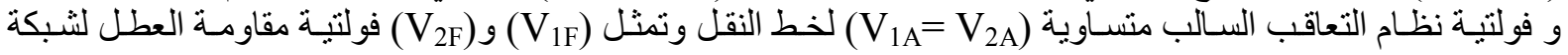

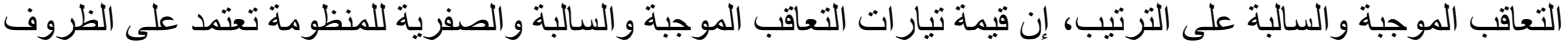

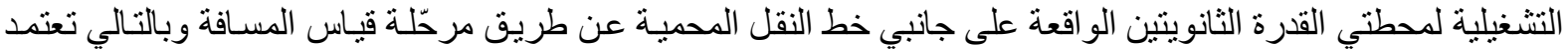
على قيمة ممانعات التعاقب الموجبة (Z)

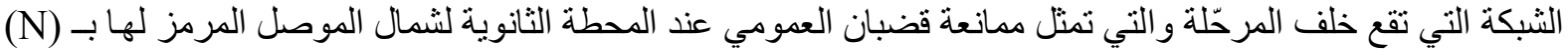

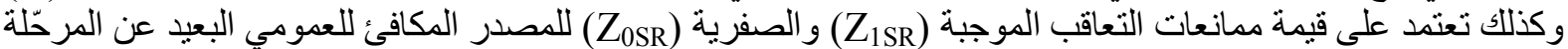

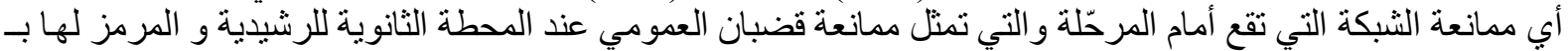

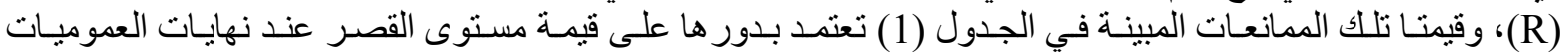

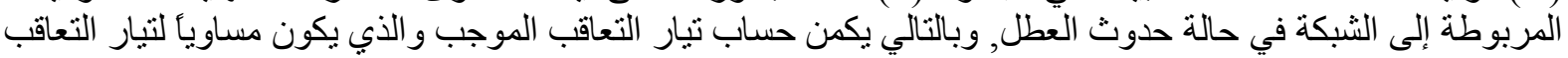

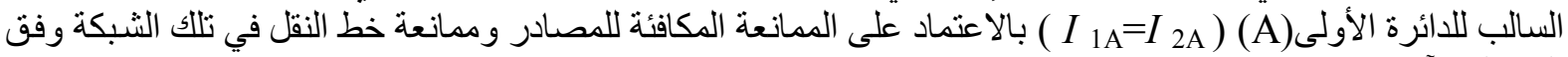

$$
I_{1 \mathrm{~A}}=I_{2 \mathrm{~A}}=\frac{(1-m) \cdot Z_{1 L}+Z_{1 S R}}{Z_{1 S N}+Z_{1 S R}+Z_{1 L}} I_{1}
$$

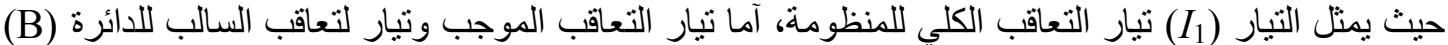

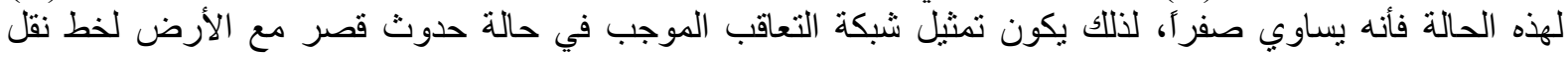


مـزدوج الدائرة في حالـة كون احد مساريه عطل و المسار الأخر خارج الخدمة و مؤرض من كلا نهايته وكما مبين في

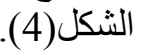

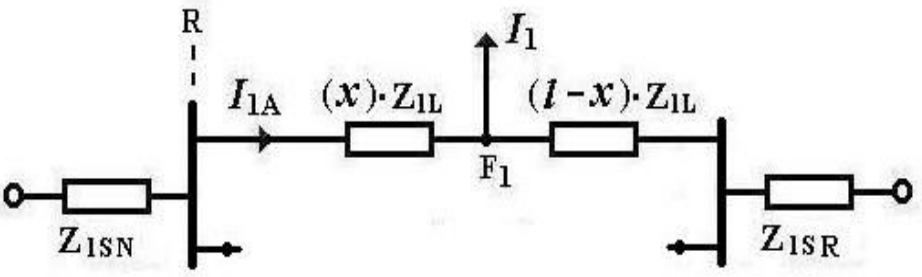

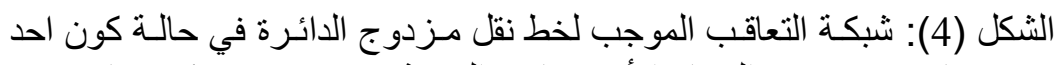

مساريه مقصور و المسار الأخر خارج الخدمة و مؤرض من كن كلا نهايته

أن نأثير الحث التبادلي للممانعة الاقتران (Z) سيظهر في شبكة التعاقب الصفري بين تيار التعاقب الصفري

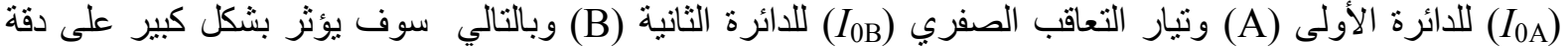

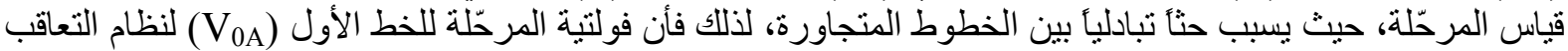

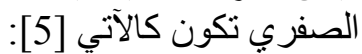

$$
\mathrm{V}_{0 \mathrm{~A}}=\mathrm{X} \cdot\left(\mathrm{Z}_{0 \mathrm{~L}} I_{0 \mathrm{~A}}+\mathrm{Z}_{0 \mathrm{M}} I_{0 \mathrm{~B}}\right)+\mathrm{V}_{0 \mathrm{~F}}
$$

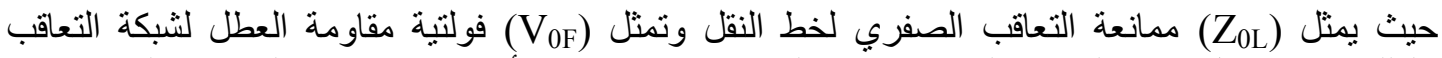
الصفري، ولذلك يكون تمثيل شبكة التعاقب الصفري في حالة حدوث قصر مع الأرض تحت نفس الظروف فلت السابقة مبين

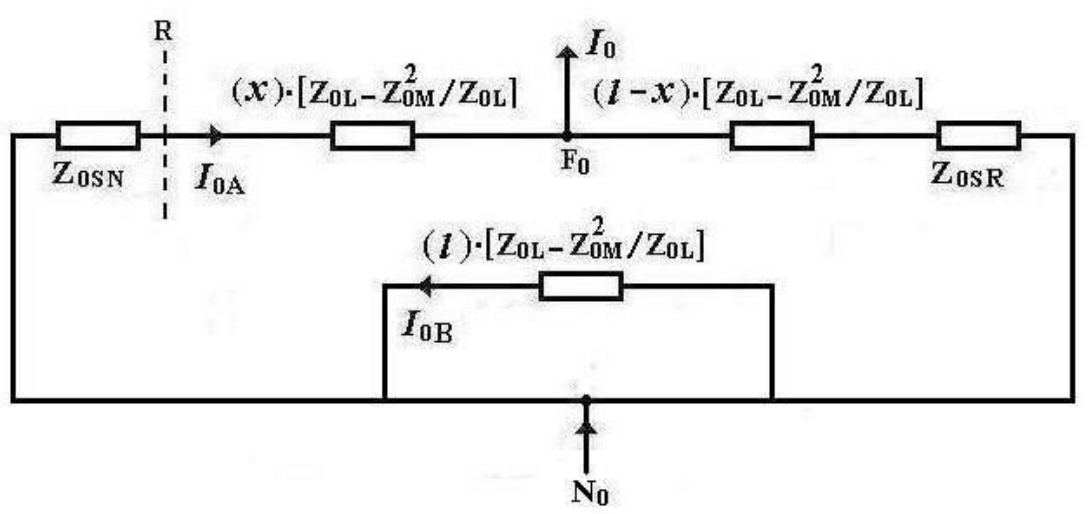

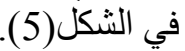

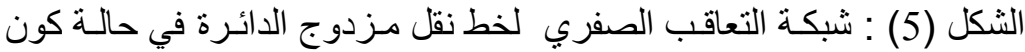

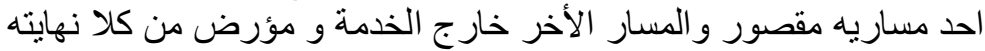

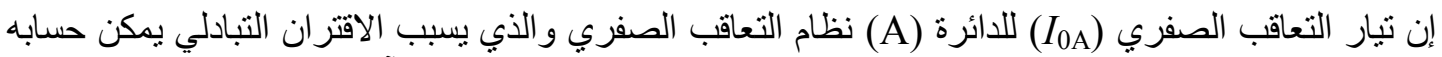

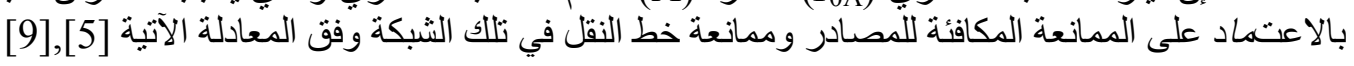

$$
I_{0 \mathrm{~A}}=\frac{(1-m) \cdot Z_{0 L}\left(1-\frac{Z_{0 M}^{2}}{Z_{0 L}^{2}}\right)+Z_{0 S R}}{Z_{0 S N}+Z_{0 S R}+Z_{0 L}\left(1-\frac{Z_{0 M}^{2}}{Z_{0 L}^{2}}\right)} I_{0}
$$

حيث تمثل (m) النسبة بعد موقع العطل إلى طول الخط الكلي، آما النسبة تبار التعاقب الصفري للائرة الأولى(A) - المسار العاطل - وتيار التعاقب الصفري للدائرة الثانية (B) - المسئ المسار المؤرض - يكون وفق العلاقة 


$$
I_{0 \mathrm{~B}}=-\frac{Z_{0 M}}{Z_{0 L}} I_{0 \mathrm{~A}}
$$

و علبه فانن الفولتية التي تظهر عبر مرحّلة قياس المسافة (VR) و التي تشمل هبوط الجهد لمركبات التعاقب الثثلاثة

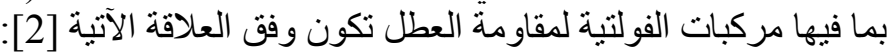

$$
\mathrm{V}_{\mathrm{R}}=\mathrm{V}_{1 \varrho 9 \mathrm{~A}}+\mathrm{V}_{2 \mathrm{~A}}+\mathrm{V}_{0 \mathrm{~A}}+\mathrm{V}_{1 \mathrm{~F}}+\mathrm{V}_{2 \mathrm{~F}}+\mathrm{V}_{0 \mathrm{~F}}
$$

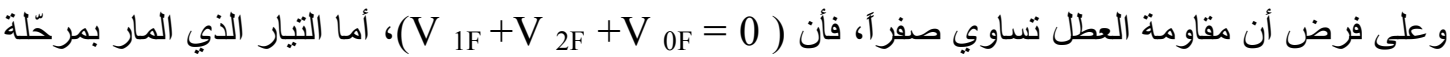
قياس المسافة (IR) مبين في المعادلة الآتية [2]:

$$
I_{\mathrm{R}}=\left(I_{1 \mathrm{~A}}+I_{2 \mathrm{~A}}+I_{0 \mathrm{~A}}\right)+\left(\frac{Z_{0 L}-Z_{1 L}}{Z_{1 L}}\right) \cdot I_{0 \mathrm{~A}}
$$

و عليه يكمن حساب الممانعة الظاهرية (ZR) التي تقيسها مرحّلة المسافة من خلال المعدلات السابقة وكالأتي

$$
Z_{R}=X\left(Z_{1 l}-\frac{I_{0 A} Z_{0 M}^{2}}{I_{R} Z_{0 L}}\right)
$$

حيث يمثل الحد الثاني من المعادلة السابقة قيمة ممانعة الخطأ بسبب ممانعة الاقتران التبادلي الناتجة من تأثثير تيار المسار المؤرض الخارج عن الخدمة و التي يمكن أن تسبب بعد في المدى (Over Reach) للقطاع المحمي لمرحّلة وقد تم استخدام برنـامج (MatlablSimulink + M-File) لتمثيل ونمذجة مرحّلة قيساس المسـافة العاملة في

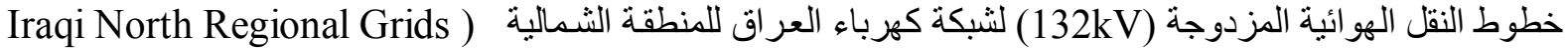
(INRG فورير (Fourier Series) لإيجاد قيم تيار ات وفولتيات التعاقب و الّنظومة فضلا لإل عن الممانعة الظاهرية المقاسة.

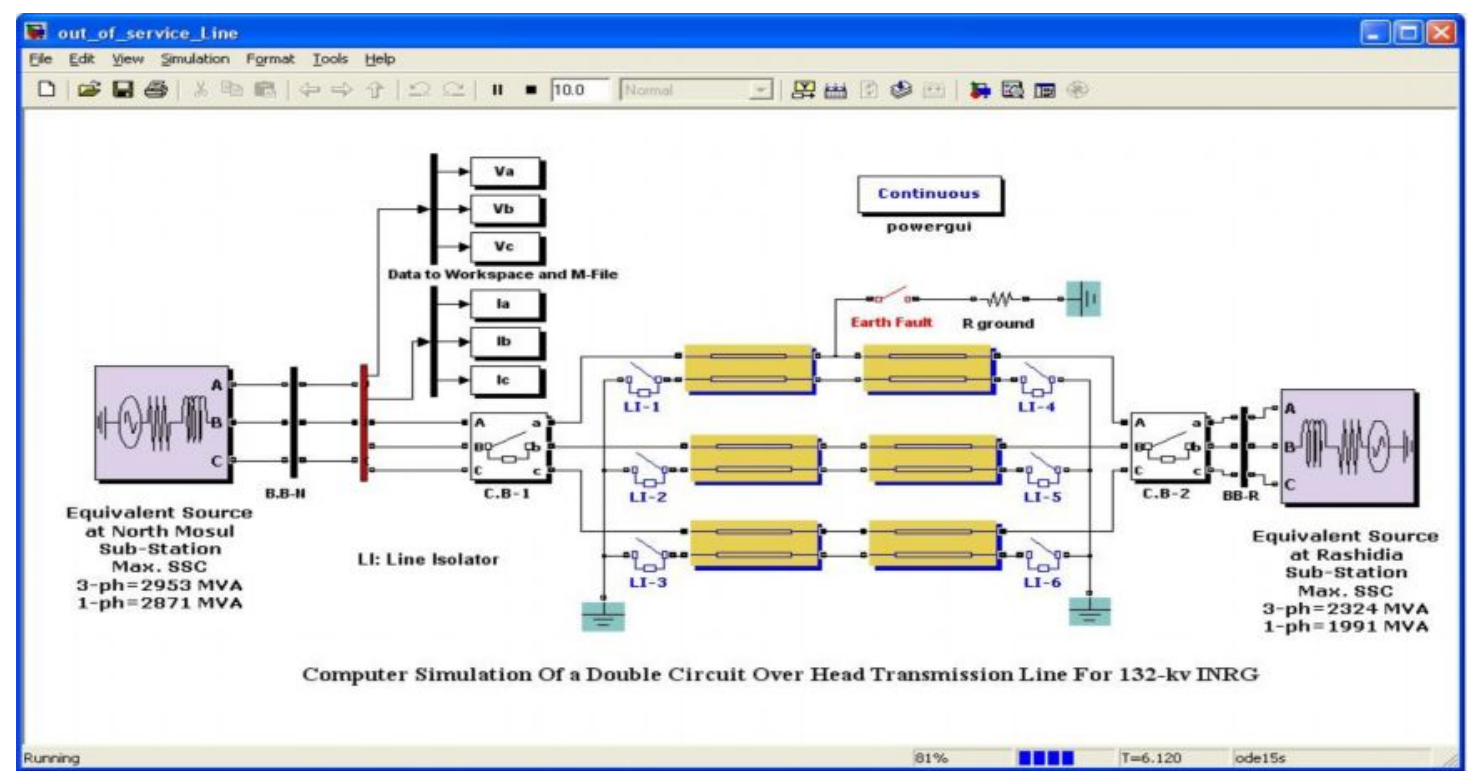

الثكل (6) 


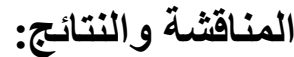

إن الحث التبادلي لنظـام التعاقب الصفري في هذه الحالـة سوف يؤنثر على قيمـة الممانعـة الظاهريـة التي تقيسهـا

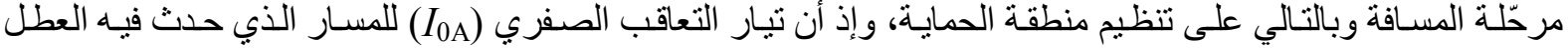

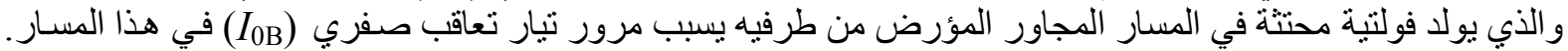

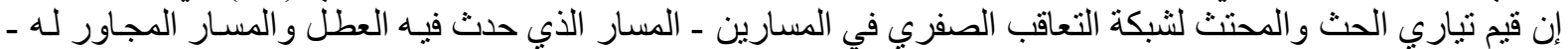

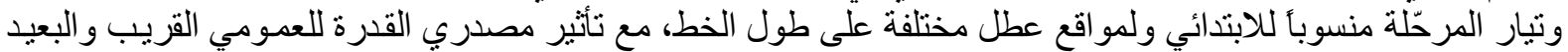

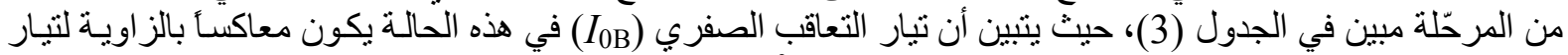

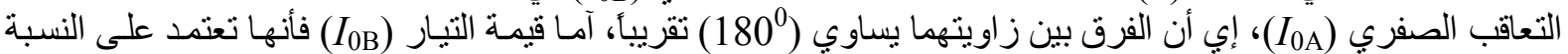

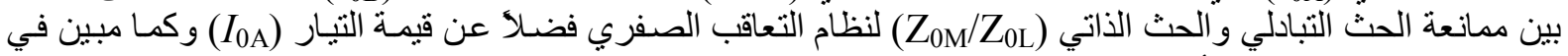

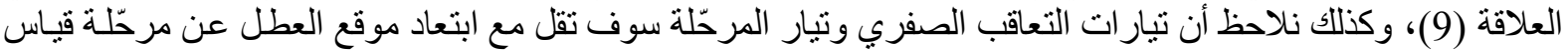

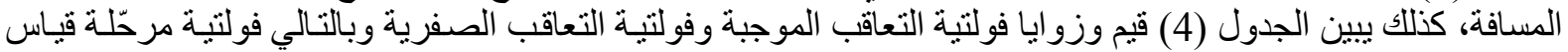

المسافة ولمو اقع مختلفة للعطل على طول خط وبفرض أن مقاومة العطل تساوي صفرًا.

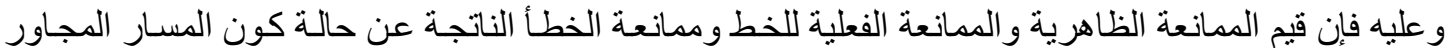

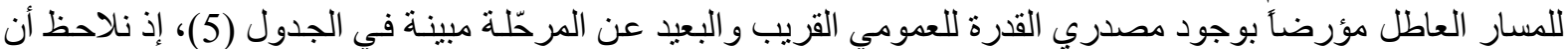

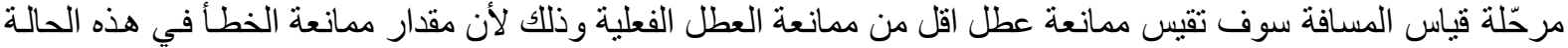

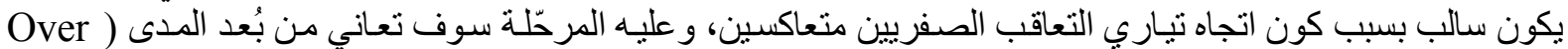
Reach

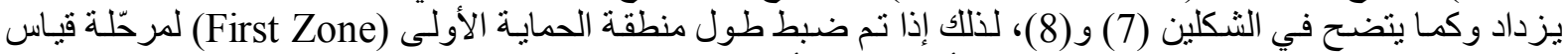

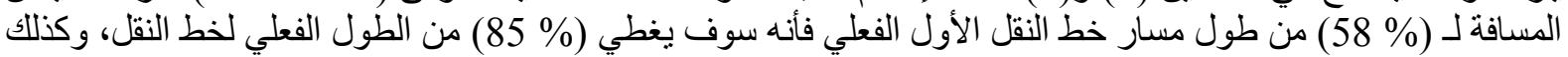

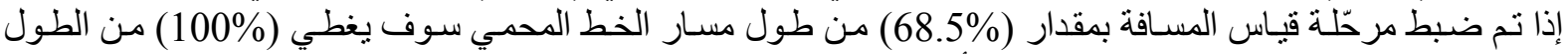

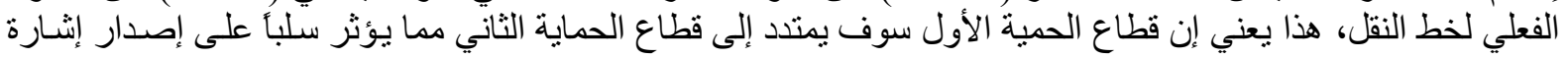
الإفلات المطلوبة للقطاع المحمي. لإن إنطي.

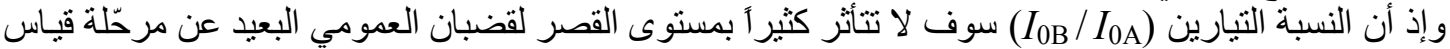

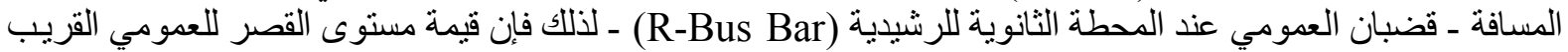

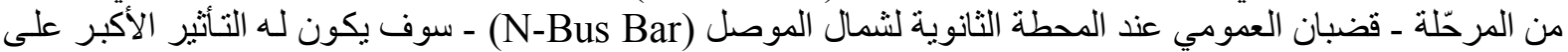
قيمة ممانعة الحث التبادلي لنظام التعاقب الصفري وكما يتضح أيضا في الثكل (7) و الثنكل (8).
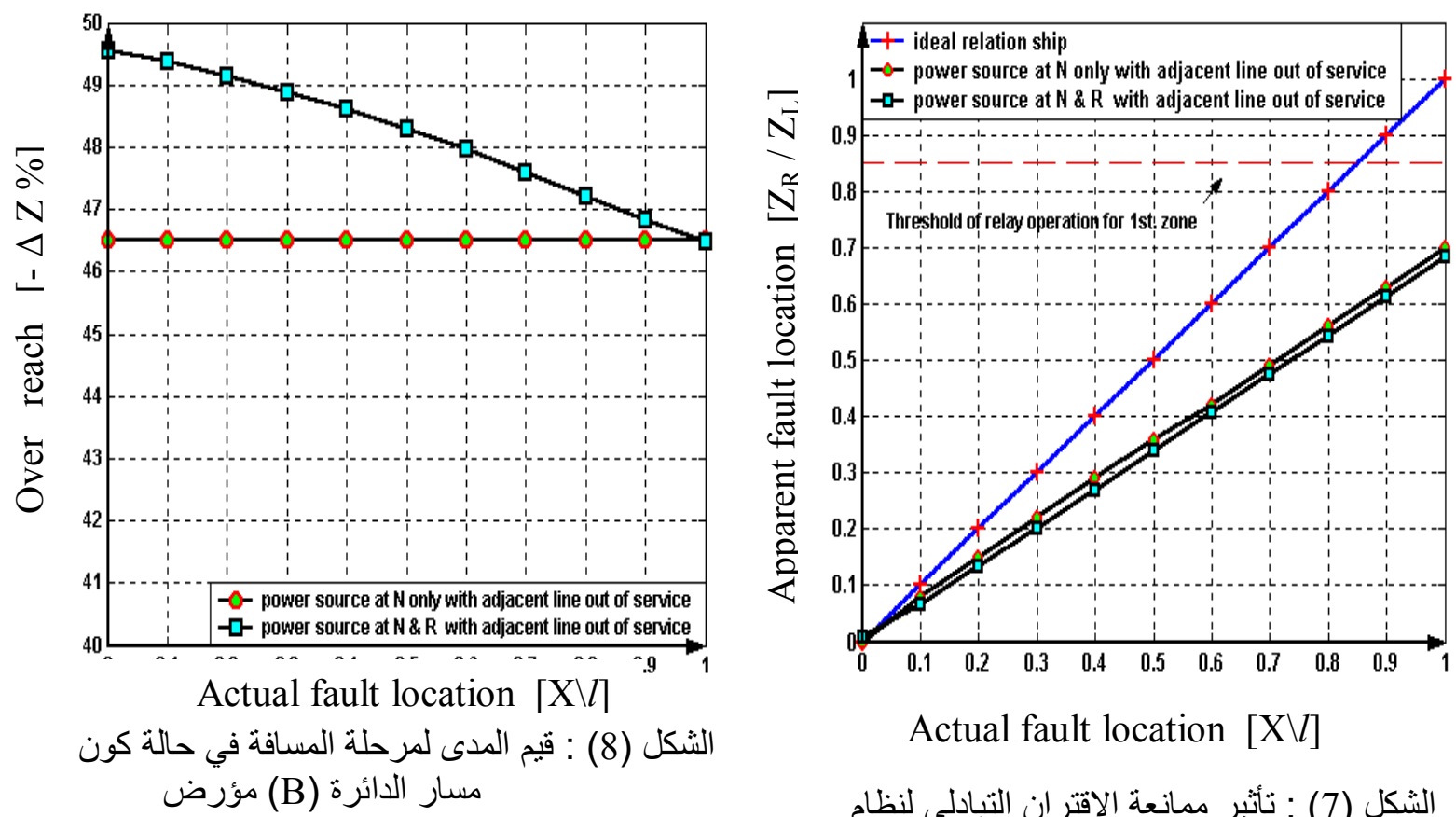

Actual fault location $[X \backslash l]$ الشكل (7) : نأثير ممانعة الاقتران التبادلي لنظام

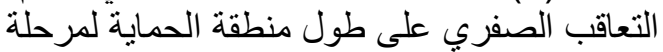

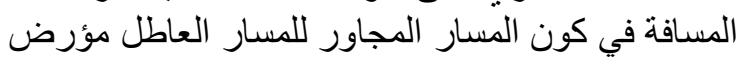




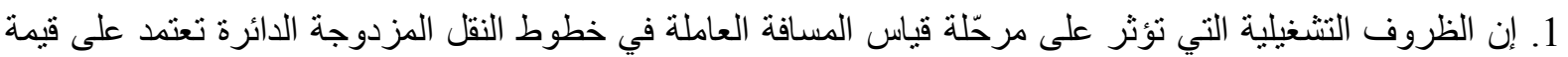

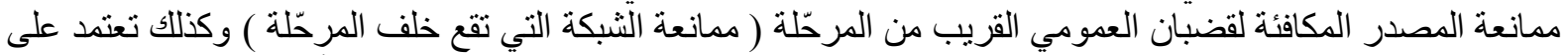

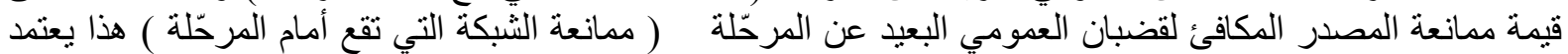

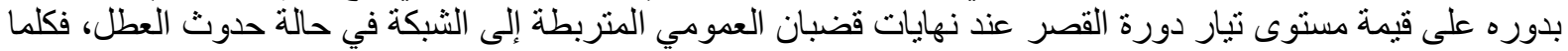

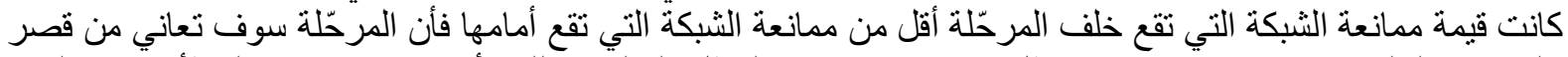

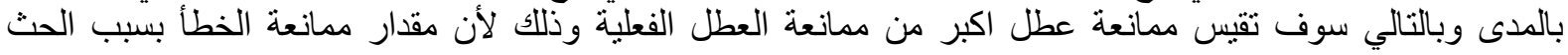

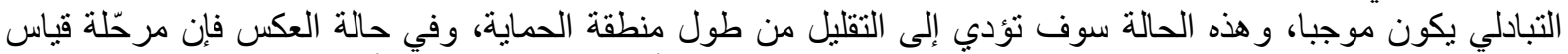

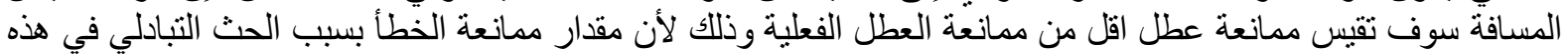

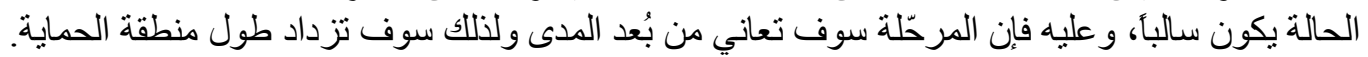

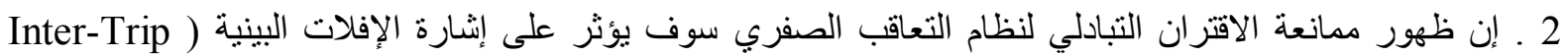

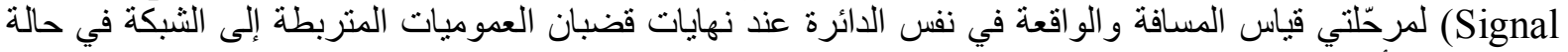

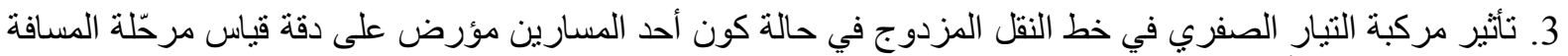

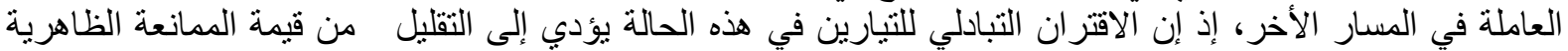

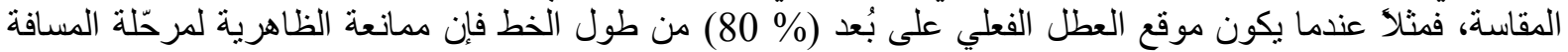

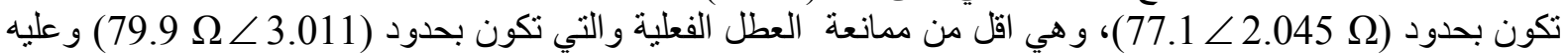

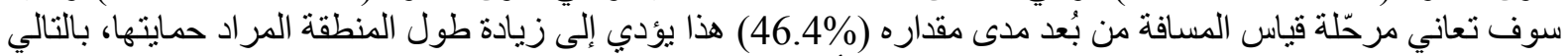

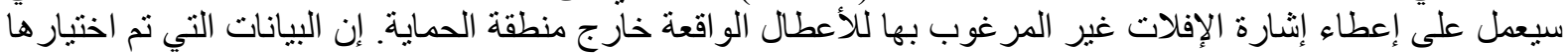

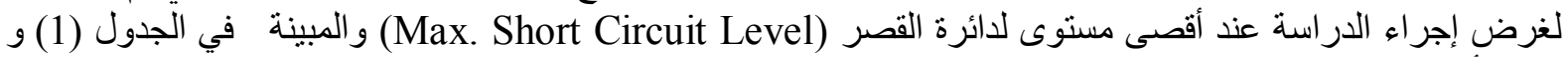
(2) مأخوذة من الثركة العامة لإنتاج الطاقة الكهربائية للمنطقة الثمالية وكانت بتاريخ 2001/12/4 و تحت الظروف

(كيلو فولت أمبير الأساس (100 MVA = (MVA base) و) (132 kV = كيلو فولت الأساس و( تردد المنظومة

$(50 \mathrm{~Hz}=$

الجدول (1) : قيم ممانعات التعاقب الموجبة ( أو السالبة ) و الصفرية المكافئة عند

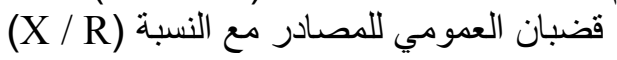

\begin{tabular}{|c|c|c|c|}
\hline $\mathrm{X} / \mathrm{R}$ & $\mathrm{Z}_{0 \mathrm{~S}}[\Omega]$ & $\mathrm{Z}_{1 \mathrm{~S}}[\Omega]$ & قبضسان العمومي \\
\hline 13.521 & $3.275+\mathrm{j} 5.959$ & $0.435+\mathrm{j} 5.889$ & موصل الشمالية \\
\hline 10.091 & $3.763+\mathrm{j} 10.81$ & $0.731+\mathrm{j} 7.457$ & الرشيدية \\
\hline
\end{tabular}

الجدول (2): قيم التيار ات لحالة القصر الثلاثي الطور وحالة قصر طور مع الأرض القاتئ بنظام الو حدة مع قيم متسو يات القصر (MAV) ) لهاتين الحالتين

\begin{tabular}{|c|c|c|c|c|}
\hline$\phi$ MVA- 1 & $\phi$ MVA- 3 & $I_{\mathrm{SC}-1} \phi[\mathrm{p} . \mathrm{u}]$ & $\boldsymbol{I}_{\mathrm{SC}-3} \phi[$ p.u $]$ & قبضـان العمومي \\
\hline 2871 & 2953 & $-76.8 \angle 28.713$ & $-85.7 \angle 29.532$ & موصل الثمالية \\
\hline 1991 & 2324 & $-78.4 \angle 19.908$ & $-84.3 \angle 23.235$ & الرشيدية \\
\hline
\end{tabular}

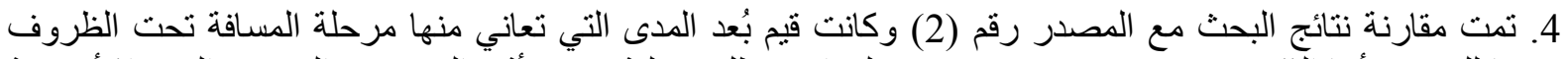
نفسها للمصدر أنفا الذكر بحدود ( 19 - 19 \% 35) ولمو اقع عطل مختلفة تحت تأثير العموميين القريب و البعيد إذ أن قيمة 


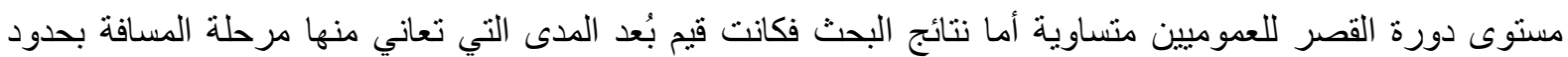

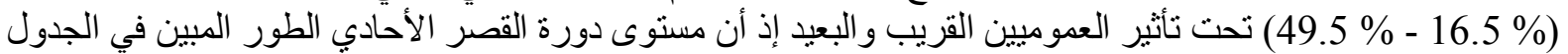

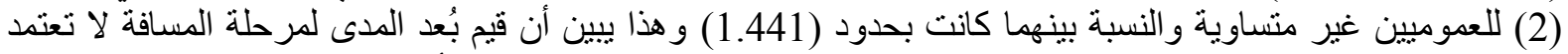

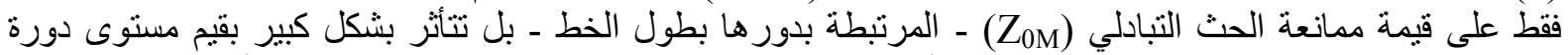

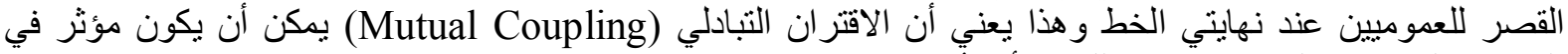
الخطوط القصيرة و المتوسطة نسبيا للسبب أعلاهي.

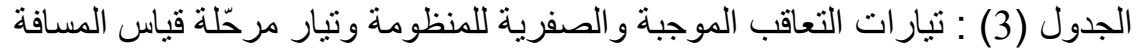

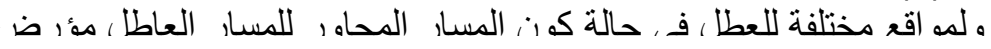

\begin{tabular}{|c|c|c|c|}
\hline تلاربتدائي [KAلة بالنسبة & 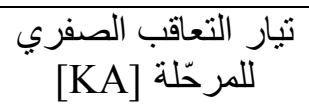 & تيار التعاقب الموجب [KA] & لموقع العطلة المئوية \\
\hline$-77.5 \angle 23.079$ & $108.9 \angle 2.587$ & $-74.1 \angle 3.789$ & $\mathrm{~m}=20 \%$ \\
\hline$-77.7 \angle 19.837$ & $108.7 \angle 2.207$ & $-74.3<3.230$ & $\mathrm{~m}=40 \%$ \\
\hline$-77.8 \angle 17.340$ & $108.6 \angle 1.912$ & $-74.4 \angle 2.798$ & $\mathrm{~m}=60 \%$ \\
\hline$-77.9<15.445$ & $108.1 \angle 1.684$ & $-74.9<2.465$ & $\mathrm{~m}=80 \%$ \\
\hline$-78.2 \angle 13.881$ & $107.7 \angle 1.492$ & $-75.3 \angle 2.183$ & $\mathrm{~m}=100 \%$ \\
\hline
\end{tabular}

الجدول (4) : فولتيات التعاقب الموجبة و الصفرية للمنظومة وفولتية مرحّلة قياس المسافة

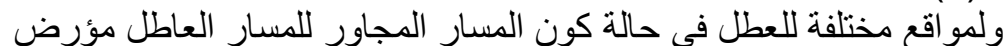

\begin{tabular}{|c|c|c|c|}
\hline فولتية المرحلة بالنسبة & فولتية التعاقب الصفرية & فولتية التعاقب الموجبة & لموقع العطلٍ المئة \\
\hline$-0.4 \angle 11.685$ & $-2.5 \angle 06.546$ & $2.4 \angle 2.538$ & $\mathrm{~m}=20 \%$ \\
\hline$-0.7 \angle 20.157$ & $-2.6<11.210$ & $2.6 \angle 4.459$ & $\mathrm{~m}=40 \%$ \\
\hline$-0.8<26.539$ & $-2.9<14.640$ & $2.7 \angle 6.001$ & $\mathrm{~m}=60 \%$ \\
\hline$-0.7 \angle 31.679$ & $-3.4 \angle 17.165$ & $2.5 \angle 7.292$ & $\mathrm{~m}=80 \%$ \\
\hline$-0.8<35.820$ & $-3.9<18.943$ & $2.6 \angle 8.421$ & $\mathrm{~m}=100 \%$ \\
\hline
\end{tabular}

الجدول (5) : ناتثير نتأريض المسار المجاور للمسار العطل على الممانعة الظاهرية المقاسة من قبل مرحّلة قياس المسافة ولمو اقع عطل مختلفة

\begin{tabular}{|c|c|c|c|}
\hline للعطل [ـمانعة الخطـاً & 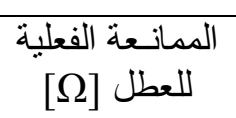 & 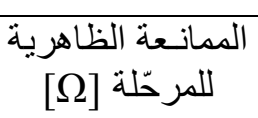 & لموقع العطلة المئوية \\
\hline$-94.1 \angle 0.248$ & $76.9 \angle 0.752$ & $77.1 \angle 0.504$ & $\mathrm{~m}=20 \%$ \\
\hline$-94.2 \angle 0.493$ & $76.9 \angle 1.505$ & $77.1 \angle 1.013$ & $\mathrm{~m}=40 \%$ \\
\hline$-94.3 \angle 0.733$ & $76.9 \angle 2.258$ & $77.1 \angle 1.526$ & $\mathrm{~m}=60 \%$ \\
\hline$-94.5<0.967$ & $76.9 \angle 3.011$ & $77.1 \angle 2.045$ & $\mathrm{~m}=80 \%$ \\
\hline$-94.7 \angle 1.191$ & $76.9 \angle 3.764$ & $77.1 \angle 2.574$ & $\mathrm{~m}=100 \%$ \\
\hline
\end{tabular}


[1] Tahahiro Kase , Phil G. Beaumont, “ Zero Sequence Current Compensation for distance Protection applied to Series Compensation Parallel Lines", Study Committee Colloquium, Calgary, CANADA, 14-16 September 2005.

[2] Gerhard Ziegler, " Numerical Distance Protection, Principles and Application " , Siemens, Erlangen, Publics -MCD-Verl. 2008.

[3] A. Abdul Ghafoor, A. Al-Badrani, "Influence of Mutual Coupling due to Zero-Sequence On The Performance of Distance Relay", Al-Rafidain Engineering Journal, Mosul University, Vol 15,No 1, June,2007.

[4] M. Sanaye, H. Seyedi, "Simulation, Analysis and Setting of Distance Relays on Double Circuit Transmission Lines", Electrical and computer Engineering Department, Faculty of Engineering, University of Tehran, 2001.

[5] ALSTOM, "Protection and Automation Net Work", Guide, Alstom T\&D Energy Automation and Information, Peter Rush, Levauios France, 2002.

[6] M. M. Saha, D. Novosel, Y. Hu and V. Leitloff," An Adaptive Scheme for parallel line distance protection ", IEEE Trans. on Power Delivery, Vol. 17, No. 1, pp. 105-11, January, 2002.

[7] S.G. Srivani, Chandrasekhar Reddy Atla, K.P.Vittal, "Comparative Evaluation of Adaptive and Conventional Distance Relay for Parallel Transmission Line with Mutual Coupling", International Journal of Mathematical, Physical and Engineering Sciences 3:2, 2009.

[8] A. Jain, A. S. Thoke, R. N. Patel,"Classification of Single Line to Ground Faults on Double Circuit Transmission Line using ANN", International Journal of Computer and Electrical Engineering, Vol. 1, No. 2, June 2009.

[9] L.Hulka, U. Klapper," Measurement of Line Impedance and Mutual Coupling of Parallel Lines to Improve the Protection System", 20th International Conference on Electricity Distribution Prague, June 2009

$$
\text { تم اجراء البحث في كلية ألهندسة = جامعة ألموصل }
$$

